\title{
Primary spleen lymphoma visualized with contrast enhanced ultrasound - Case report
}

\author{
Joanna Kurzepa', Sebastian Uhlig' , Elżbieta Czekajska-Chehab', Elżbieta Siek', \\ Magdalena Zakościelna', Andrzej Drop ${ }^{1}$ \\ ${ }^{1}$ Ist Department of Medical Radiology, Medical University of Lublin
}

Kurzepa J, Uhlig S, Czekajska-Chehab E, Siek E, Zakościelna M, Drop A. Primary spleen lymphoma visualized with contrast enhanced ultrasound - Case report. J Pre-Clin Clin Res. 2017; 11(2): 157-159. doi: 10.26444/jpccr/80919

\begin{abstract}
Introduction. The case is reported of an extremely rare primary splenic lymphoma (PSL) and summarizes the basic concepts and techniques of contrast enhanced ultrasonography (CEUS), focusing on splenic applications for the diagnosis of malignant splenic lesions.

Case report. A 52-year-old male with no co-morbidities presented with a history of left upper abdominal pain for one month, and chronic fatigue. Abdominal ultrasound was performed in which suspicious focal lesions in the enlarged spleen was detected, and assessed with contrast enhanced ultrasound, followed by magnetic resonance imaging (MRI). The diagnosis of malignant splenic tumour was made based on history, clinical examinations and radiographic appearance in CEUS and MRI.

Conclusion. In the presented case contrast enhanced ultrasound allowed establishing the diagnosis of malignancy within the spleen, which was later confirmed with MRI and histopathological examination.
\end{abstract}

\section{Key words}

contrast enhanced ultrasonography, CEUS, Primary splenic lymphoma, PSL

\section{INTRODUCTION}

Primary splenic lymphomas (PSL) are extremely rare $(<1 \%$ of all lymphomas) and usually represent non-Hodgkin lymphomas of B-cell origin. More often the spleen is involved by lymphoma as a part of a systemic illness (30-40\% of cases of systemic lymphoma, in both Hodgkin's and nonHodgkin's disease) with enlarged abdominal lymph nodes [1-3].

Ultrasonography is routinely the first-line diagnostic procedure in the detection of focal splenic lesions. Realtime contrast-enhanced sonography improves detection of splenic pathology, and can provide useful information for differential diagnosis between benign and malignant focal splenic lesions.

The case is reported of an extremely rare primary splenic lymphoma, and summarizes the basic concepts and techniques of contrast enhanced ultrasonography (CEUS), focusing on splenic applications for the diagnosis of malignant splenic lesions.

\section{CASE REPORT}

A 52-year-old male with no co-morbidities presented with a history of left upper abdominal pain for one month, and chronic fatigue. Physical examination and initial laboratory tests revealed no specific abnormalities. Abdominal ultrasound in B-mode with Power Doppler, CEUS and MRI were performed. Diagnosis of primary tumour of the spleen was made and the patient posted for splenectomy.

Address for correspondence: Joanna Kurzepa, Ist Department of Medical Radiology Medical University of Lublin, Jaczewskiego 8, 20-954 Lublin, Poland

E-mail: kurzepa.joanna@gmail.com

Received: 10 November 2017; accepted: 30 November 2017

\section{RESULTS}

Ultrasound examination (US) was performed using GE system (GE Healthcare, Buc, France) with a convex transducer (frequency 3,5MHz, mechanical index). The study protocol included B-mode presentation, Power Doppler imaging and CEUS.

Acquisition using B-mode presentation included morphological assessment of the spleen and focused on splenic lesion. US showed splenomegaly and two solid mass tumors within the spleen: a larger hypoechoic focal lesion located near the splenic hilum, and an accompanying smaller lesion with small cystic compound (Fig. 1). There was no generalized lymphadenopathy. These results did not indicate the presence of metastatic tumours.

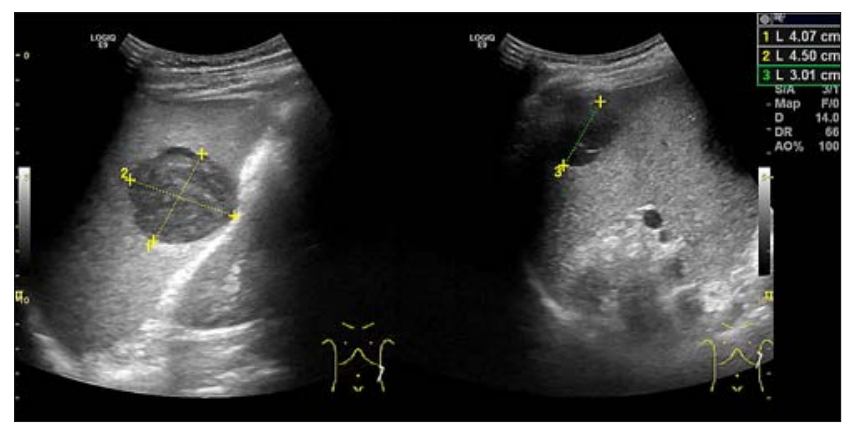

Figure 1. Focal lesions in the spleen detected in B-mode US

On Power Doppler sonography, the vascularity and patterns of blood flow within and around tumours were assessed, using the surrounding splenic tissue as an in vivo reference. The lesions showed intense peripheral and weaker, chaotic internal vascularization (Fig. 2).

The larger tumour was chosen as the target lesion for further assessment with CEUS which was performed in 


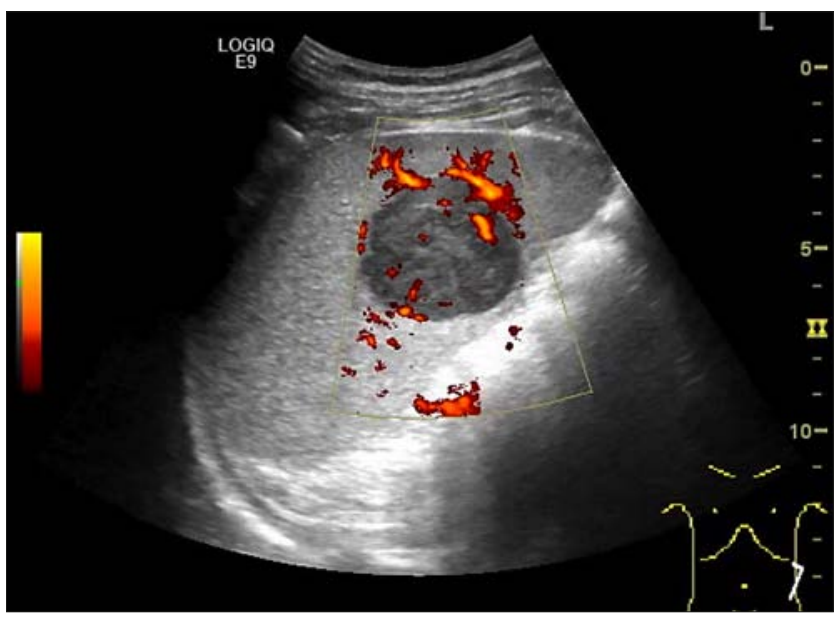

Figure 2. Irregular vascularization of target lesion presented in PD

harmonic mode with a low mechanical index. A $2 \mathrm{ml}$ bolus of second-generation contrast medium (Sonovue-Bracco Int., Milan, Italy) was administered into the antecubital vein, followed by $10 \mathrm{ml}$ of a $0.9 \%$ saline bolus. CEUS study was analyzed by scanning from $0-50$ seconds after injection during the arterial phase, between 50-120 seconds during the parenchymal phase, and between 120 seconds and 4 minutes after injection during the late phase. On CEUS, the target lesion revealed a rapid influx of the contrast agent, resulting in homogenous enhancement similar to the surrounding splenic tissue in the arterial phase. In the early stages of the arterial phase, it started to show rapid and intense wash-out which was intensified in parenchymal and late phases, eventually revealing a inhomogeneous, hypoechoic ultrasound structure (Fig. 3, 4). Due to limitations of CEUS,

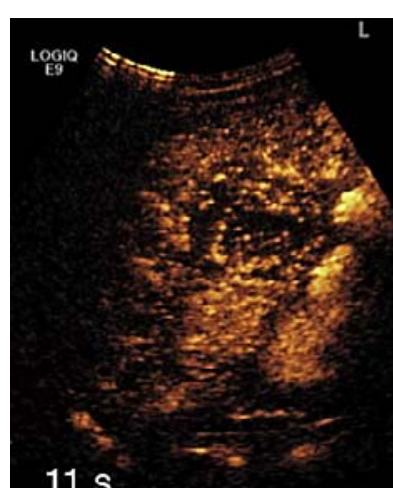

$11 \mathrm{~s}$.
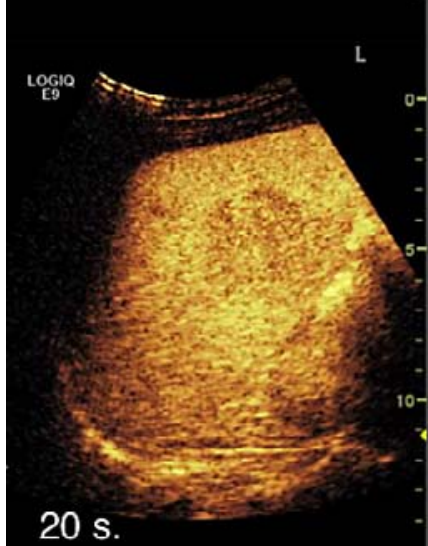

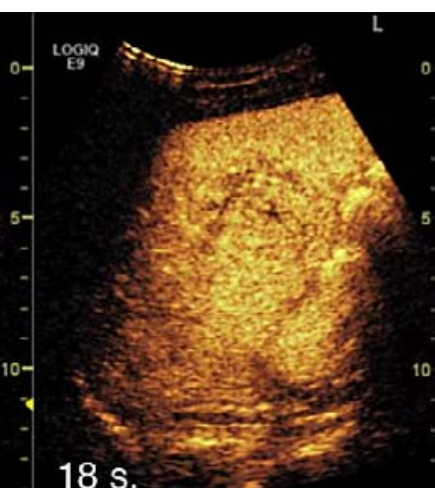

$18 \mathrm{~s}$.

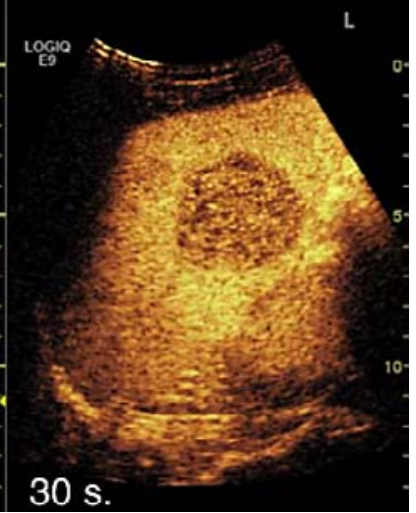

Figure 3. Pattern of enhancement of target lesion in arterial phase of CEUS examination in $11,18,20$ and 30 seconds after contrast administration

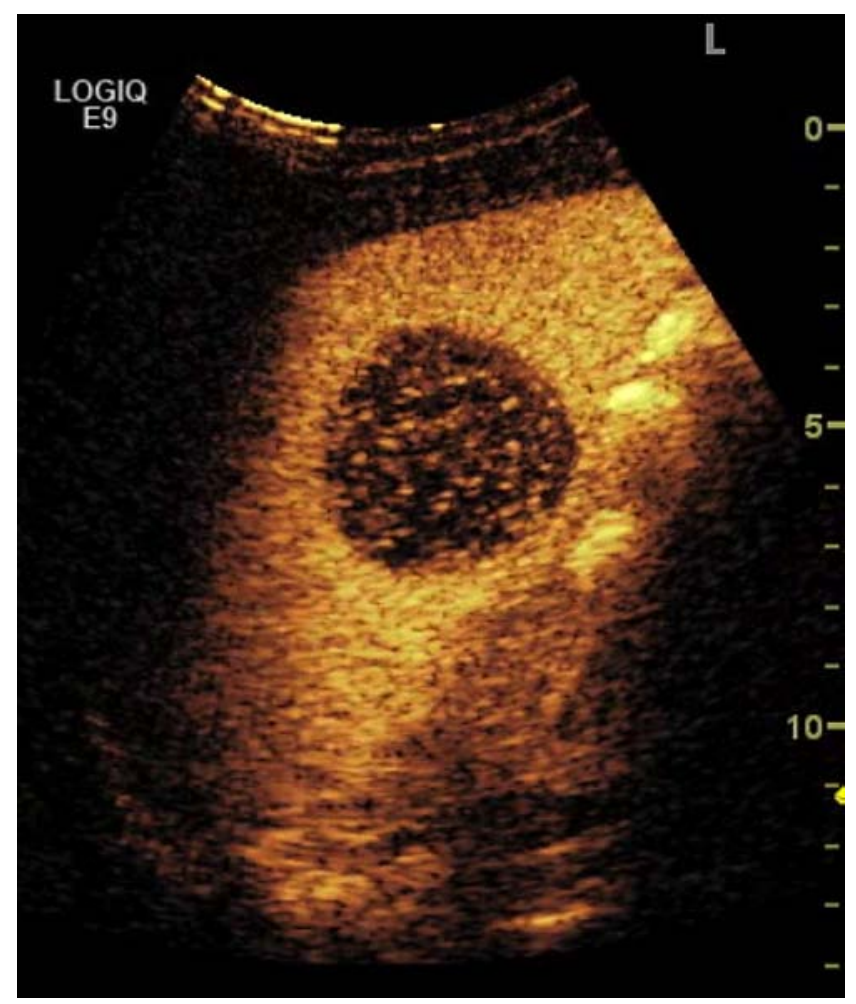

Figure 4. Intense washout in late phase (120 s.)

the second lesion was assessed in parenchymal and late phases only, presenting a similar appearance to the target lesion.

MRI confirmed the ultrasound diagnosis of primary malignant tumour of the spleen.

The patient underwent open splenectomy. Histopathological examination made a diagnosis of splenic Non-Hodgkin's Lymphoma of the B cell origin.

\section{DISCUSSION}

The spleen is particularly suitable for CEUS studies because of its superficial location, small size, homogeneous parenchyma, high vascularization, and tendency to retain contrast microbubbles longer than other organs (long-lasting and intense contrast enhancement) [4-6]. At the present time, the European Guidelines for nonhepatic applications of CEUS warrant the use of this tool for investigation of the spleen in four cases [6]:

1) to characterize splenic parenchymal inhomogeneity or suspected lesions on conventional US;

2) to confirm suspected splenic infarction;

3) to characterize suspected accessory spleens or splenosis;

4) to detect splenic malignant lesions in oncologic patients when CT and/or MRI and PET are contraindicated or inconclusive.

Additionally, CEUS can be employed in abdominal trauma imaging, including splenic injuries, especially as an alternative to CT in stable patients, and for the follow-up to avoid CT radiation exposure [6].

The diagnostic value of CE-sonography in differentiating benign from malignant splenic focal lesions is based on different dynamics of uptake and washout of contrast 
agent during arterial and parenchymal phase [2]. There are essentially three enhancement patterns of splenic lesions $[6,7]$ :

1) Lesions without enhancement at any phases. This pattern is typical for benign pathologies, such as simple cyst, splenic infarct, haematoma, sarcoid granulomas, bacterial abscess [7].

2) Lesions that enhance in the arterial phase followed by persistent enhancement in the parenchymal and late phase, or with late phase washout. It can be observed typically in splenic haemangiomas, hamartoma $[7,8]$. The great intensity of early arterial enhancement in benign splenic pathologies reflects hypervascularity caused by an increased arterial blood flow [2].

3) Lesions with arterial enhancement and rapidly washout, a typical pattern for malignant lesions, such as metastases or lymphoma. In the arterial phase malignant focal splenic lesions typically present heterogenous or homogenous hypo-enhancement. Reduced arterial enhancement in this case is probably due to neoangiogenesis, which creates a poor and slow flow $[2,7]$.

The most important feature differentiating the benign from malignant washout is related to timing. Generally, malignant lesions washout markedly and rapidly while benign shows later, more gradual washout $[6,7]$.

Primary malignant tumours of the spleen are very rare. The most common include: lymphoid neoplasms, arising from the white pulp and vascular neoplasms which arise from the red pulp [9]. Ultrasonography is routinely the first-line diagnostic procedure in the detection of focal splenic lesions. Saboo et al. described four imaging patterns of splenic involvement in primary and secondary lymphoma. The most common one is the homogeneous splenomegaly (without a focal lesion), which indicates diffuse infiltration of the spleen. Splenic lymphoma can also appear on the grey scale as small, focal or miliary nodules (less than $5 \mathrm{~mm}$ ), multiple large nodular lesions and bulky solid masses $[1,10]$. Focal lesions are most often hypoechoic relative to normal splenic parenchyma $(60-70 \%)[2,7]$. Hiperechogenicity is not typical feature for splenic lymphoma and require histological confirmation. Lesions larger than $3 \mathrm{~cm}$ may present as cyst-like appearance with an indistinct boundary echo pattern typical for highgrade non-Hodgkin lymphoma [11]. Rare US features include nodules with a 'target sign' (15.4\%), a heterogenous, complex pattern which is typical of the lesions after chemotherapy, and internal calcifications $[2-4,12]$. The normal size and US appearance of the spleen does not exclude the lymphoma $[3,11]$.

In most cases, CEUS allows better demarcation of a lymphoid lesion from the surrounding splenic tissue [8, 11]. In arterial the phase, splenic lymphoma appears most commonly as hypoenhancing (50-53\%) or isoenhancing (37.5-46.35\%), compared to the rest of the spleen, although rare hyperenchancing lesions have also been described [2, 13]. Occasionally, lymphomatous lesions may display a small, irregular rim of enhancement during the early arterial phase $[13,14]$. In the parenchymal phase, the lesions become more conspicuous and progressively more hypoenhancing due to rapid washout [2, 7]. 120-180 seconds after IV contrast injection, splenic lymphoma lesions characterize no detectable enhancement [13]. Rarely, late phase imaging can demonstrate subtle enhancing regions corresponding to microcirculation within the tumour tissue that may not be seen during the arterial phase $[4,7]$.

\section{CONCLUSION}

In the presented case, contrast enhanced ultrasound allowed establishing the diagnosis of malignancy within the spleen, which was later confirmed with MRI and histopathological examination.

\section{REFERENCES}

1. Kaza RK, Azar S, Al-Hawary MM, Francis IR. Primary and secondary neoplasms of the spleen. Cancer Imaging. 2010; 10: 173-82.

2. Caremani M, Occhini U, Caremani A, Tacconi D, Lapini L, Accorsi A, et al. Focal splenic lesions: US findings. J Ultrasound. 2013; 16: 65-74.

3. Vancauwenberghe T, Snoeckx A, Vanbeckevoort D, Dymarkowski S, Vanhoenacker FM. Imaging of the spleen: what the clinician needs to know. Singapore Med J. 2015; 56: 133-44.

4. Catalano O, Lobianco R, Sandomenico F, D’Elia G, Siani A. Real-time contrast-enhanced ultrasound of the spleen: examination technique and preliminary clinical experience. Radiol Med. 2003; 106: 338-56.

5. Chiavaroli R, Grima P, Tundo P. Characterization of nontraumatic focal splenic lesions using contrast-enhanced sonography. Journal of Clinical Ultrasound. 2011; 39: 310-5.

6. Piscaglia F, Nolsoe C, Dietrich CF, Cosgrove DO, Gilja OH, Bachmann Nielsen $M$, et al. The EFSUMB Guidelines and Recommendations on the Clinical Practice of Contrast Enhanced Ultrasound (CEUS): update 2011 on non-hepatic applications. Ultraschall Med. 2012; 33: 33-59.

7.Sutherland T, Temple F, Galvin A, Hennessy O. Contrast-enhanced ultrasound of the spleen: an introduction and pictorial essay. Insights Imaging. 2011; 2: 515-24.

8. Hopkins A CD, Antypa EG, Piperopolus PN. Contrast enhanced sonographic study of the spleen. Hellenic Journal of Radiology. 2017; 2: 49-65.

9. Dachman AH FA. Radiology of the spleen. St. Louis, MO: Mosby; 1993.

10. Saboo SS, Krajewski KM, O’Regan KN, Giardino A, Brown JR, Ramaiya $\mathrm{N}$, et al. Spleen in haematological malignancies: spectrum of imaging findings. Br J Radiol. 2012; 85: 81-92.

11. Chiorean L, Zdrenghea M, Badea R. Ultrasonography of the spleen. Pictorial essay. Med Ultrason. 2014; 16: 48-59.

12. Wan YL, Cheung YC, Lui KW, Tseng JH, Lee TY. Ultrasonographic findings and differentiation of benign and malignant focal splenic lesions. Postgrad Med J. 2000; 76: 488-93.

13. von Herbay A, Barreiros AP, Ignee A, Westendorff J, Gregor M, Galle PR, et al. Contrast-enhanced ultrasonography with SonoVue: differentiation between benign and malignant lesions of the spleen. J Ultrasound Med. 2009; 28: 421-34.

14. Luna A VJ, da Cruz LC Jr., Rossi SE. Functional Imaging in Oncology. Clinical Applications. Berlin, Heidelberg: Springer; 2014. 